\title{
Voltage phase angle jump characteristic of DFIGs in case of weak grid connection and grid fault
}

\author{
Xinshou TIAN ${ }^{1,2}$, Gengyin $\mathrm{LI}^{1}$, Yongning $\mathrm{CHI}^{2}$, Weisheng WANG ${ }^{2}$, \\ Haiyan TANG ${ }^{2}$, Xiang $\mathrm{LI}^{2}$
}

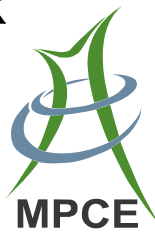

\begin{abstract}
In the condition of connecting large scale doubly-fed induction generators (DFIGs) into weak grid, the closely coupled interactions between wind generators and power grid becomes more severe. Some new fault characteristics including voltage phase angle jump will emerge, which will influence the power quality of power system. However, there are very few studies focusing on the mechanism of voltage phase angle jump under grid fault in a weak grid with wind turbine integration. This paper focuses on the scientific issues and carries out mechanism studies from different aspects, including mathematical deduction, field data analysis and time domain simulation. Based on the analysis of transient
\end{abstract}

CrossCheck date: 16 December 2015

Received: 24 August 2015/Accepted: 21 December 2015/

Published online: 18 February 2016

(C) The Author(s) 2016. This article is published with open access at Springerlink.com

$\triangle$ Xinshou TIAN

tianxinshou@epri.sgcc.com.cn

Gengyin LI

ligy@ncepu.edu.cn

Yongning $\mathrm{CHI}$

chiyn@epri.sgcc.com.cn

Weisheng WANG

wangws@epri.sgcc.com.cn

Haiyan TANG

tanghy@epri.sgcc.com.cn

Xiang LI

wishx@126.com

1 State Key Laboratory of Alternate Electrical Power System with Renewable Energy Sources (North China Electric Power University), Changping District, Beijing 102206, China

2 China Electric Power Research Institute, Haidian District, Beijing 100192, China characteristics of DFIGs during the grid fault, this paper points out that the change of terminal voltage phase angle in DFIGs is an electromagnetism transition process, which is different from conventional synchronous generator. Moreover, the impact on transient characteristics of voltage phase angle are revealed in terms of fault ride through (FRT) control strategies, control parameters of current inner-loop of rotor-side converter and grid strength.

Keywords DFIGs, Weak grid, Fault ride through, Voltage phase angle jump

\section{Introduction}

Wind power has experienced a rapid development during the past 15 years, and the total installed capacity of wind power has reached $114.6 \mathrm{GW}$ by the end of 2014 in China [1]. "Centralized development and long-distance transmission" is the outstanding characteristic of wind energy development in China. Therefore, most wind farms are located at the end of the power grid, and the electrical connection between wind generators and power grid is weak. Grid disturbance will cause complex transient process of wind turbines [2]. A key problem for reliability and security of power grid is how to accurately evaluate the transient characteristics of wind turbines and its impacts on transient stability of power system when large scale wind turbines are connected into weak grid [3].

DFIGs not only improve the energy conversion efficiency greatly, but also reduce the mechanical stress of the prime mover. It could improve the control capability and stability of power system, and becomes one of the commercial wind turbines with successful operation $[4,5]$. In order to enhance the transient stability of power systems, FRT has become the basic requirements for wind farms [6]. However, when 
connecting large scale DFIGs into weak grid or micro-grid, the current DFIG control strategies which are designed based on an assumption of strong grid connection condition are very difficult to be adapted. The transient processes become more complicated, and some new fault characteristics will appear. Thus, it is a greater challenge for FRT of DFIGs [7, 8].

In recent years, the transient characteristics of power system integrating large wind power have drawn great attention to the engineers and scientists of power systems. There are three methods for analyzing the transient characteristics of DFIGs and the impacts on power system, which are theoretical analysis, test researches and time domain simulation approaches, and some meaningful conclusions have been acquired. Several promising methods for FRT based on crowbar protection circuit have been provided in [9-11]. In addition, some FRT control strategies considering rotor excitation control have been developed in $[8,12,13]$, and the fault current characteristics of DFIGs are given through deriving analytical expressions of fault current. There are also some studies focusing on the transient characteristics of wind turbines and the impacts on transient stability of power system [14-16]. However, these studies just consider the characteristic of voltage amplitude variation under grid fault. The fault current characteristics of DFIGs considering voltage phase angle jump are analyzed in [17-22], but there is no strictly theoretical analysis on the mechanism of voltage phase angle jump when a grid fault occurs in the condition of connecting large scale DFIGs into weak grid. The influencing factors are unclear, and the conclusions are controversial.

The accurate analysis on transient characteristics of power system is the basis for security and stability of power system. The transient process of DFIGs is strongly nonlinear and coupling dynamic process, especially in the condition of weak grid it is more difficult to achieve accurately analysis. The purpose of this paper is to reveal the mechanism of voltage phase angle jump and the influencing factors in the condition of connecting large scale DFIGs into weak grid. The main work of this paper can be described as follows. Section 2 discusses the mathematical model of DFIGs and the main control system. The transient process of DFIGs is described under grid fault in weak grid by mathematical deduction in Sect. 3. Section 4 analyzes the characteristics of voltage phase angle jump and its influencing factors. Section 5 carries out the experiments and discusses. In the end, conclusions are drawn in Sect. 6.

\section{Mathematical model of DFIGs and main control system}

In recent years, a lot of studies on model and controller of variable speed wind turbines have been conducted regarding power system analysis. Fifth-order mathematical model of DFIGs contains the complete dynamic behaviors of stator and rotor, and its effectiveness has been widely verified. It is widely used to analyze the transient process of DFIGs. The voltage and flux plural vector model of DFIGs in the $d q$ rotating reference frame can be expressed as follows [5].

$$
\left\{\begin{array}{l}
U_{\mathrm{s}}=\frac{\mathrm{d} \psi_{\mathrm{s}}}{\mathrm{d} t}+\mathrm{j} \omega_{\mathrm{e}} \psi_{\mathrm{s}}+R_{\mathrm{s}} I_{\mathrm{s}} \\
U_{\mathrm{r}}=\frac{\mathrm{d} \psi_{\mathrm{r}}}{\mathrm{d} t}+\mathrm{j} \omega \psi_{\mathrm{r}}+R_{\mathrm{r}} I_{\mathrm{r}} \\
\psi_{\mathrm{s}}=L_{\mathrm{s}} I_{\mathrm{s}}+L_{\mathrm{m}} I_{\mathrm{r}} \\
\psi_{\mathrm{r}}=L_{\mathrm{r}} I_{\mathrm{r}}+L_{\mathrm{m}} I_{\mathrm{s}}
\end{array}\right.
$$

where $U$ is the voltage; $I$ is the current; $\psi$ is the flux; $R$ is the resistance; $L$ is the inductance; $\omega_{\mathrm{e}}$ is the synchronous angular velocity; $\omega$ is the slip angular velocity; subscript $s$ is the stator component; subscript $r$ is the rotor component; subscript $m$ is the mutual interaction component.

Figure 1 shows the equivalent circuit of DFIGs. In Fig. $1, L_{\delta \mathrm{s}}$ is the leakage inductance of stator; $L_{\delta \mathrm{r}}$ is the leakage inductance of rotor.

The rotor current control method based on the stator voltage oriented for DFIGs is adopted in the paper, the active and reactive power output can be controlled independently through feed-forward compensation method. The control structure of current inner-loop of rotor-side converter is shown in Fig. 2 . In Fig. 2, $i_{\text {rd_ref }}, i_{\text {rq_ref }}$ are rotor current reference values; $u_{\text {rd_ref }}, u_{\text {rq_ref }}$ are rotor voltage reference values; $\delta$ is the leakage inductance coefficient of generator and $\delta=1-L_{\mathrm{m}}^{2} /\left(L_{\mathrm{s}} L_{\mathrm{r}}\right)$.

Ignoring the switch transients of converters and assuming that the actual value is equal to the reference value of rotor voltage, then the rotor voltage of DFIGs is expressed as

$U_{\mathrm{r}}=K_{\mathrm{p}} \Delta I_{\mathrm{r}}+K_{\mathrm{i}} \int \Delta I_{\mathrm{r}} \mathrm{d} t+\mathrm{j} \omega \sigma L_{\mathrm{r}} I_{\mathrm{r}}$

where $K_{\mathrm{p}}$ is the proportion control parameter; $K_{\mathrm{i}}$ is the integral control parameter; $\Delta I_{\mathrm{r}}$ is the deviation between reference value and actual value of rotor current, i.e., $\Delta I_{\mathrm{r}}=I_{\mathrm{r}_{\mathrm{r}} \text { ref }} I_{\mathrm{r}}$.

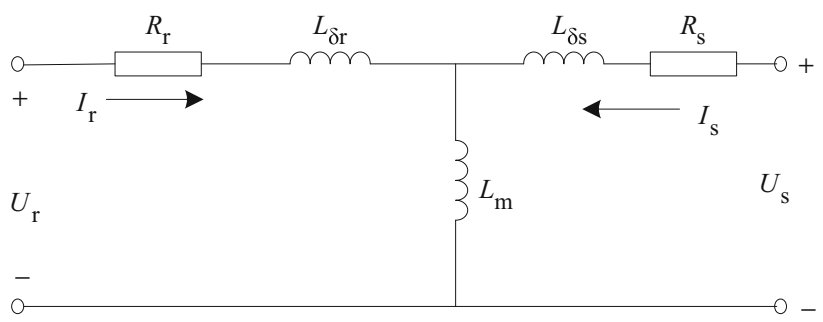

Fig. 1 Equivalent circuit of DFIGs 


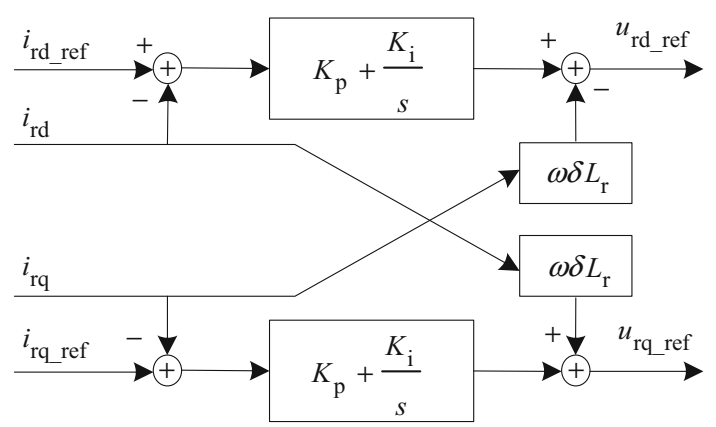

Fig. 2 Control structure of current inner-loop of rotor-side converter

\section{Analysis on transient process under grid fault in case of connecting DFIGs into weak grid}

When a grid fault occurs, the structure of power system will change, and there will be a switch from normal operation control strategy to FRT control strategy of DFIGs. If the magnetic circuit of generator is not saturated during the grid fault, the superposition principle can be used to analyze the transient process of DFIGs. The transient process can be described by summing steady-state component and transient component. The steady-state component is determined by the operating condition before grid fault, while the transient component consists of two parts. One of the transient components is the so called grid fault voltage component, which includes voltage drop and phase angle change directly caused by grid fault. The other one is the control response fault component, which is caused by the switch from normal operation control strategy to FRT control strategy of DFIGs.

The superposition principle of grid connection of large scale DFIGs under grid fault is shown in Fig. 3.

\subsection{Steady-state component of fault voltage}

The voltage, current and flux vector of DFIGs are relative rest in the $d q$ rotating reference frame, and the flux of generator is constant in the steady-state condition. The terminal voltage and fault voltage component according to Fig. $3 \mathrm{~b}$ can be formulated as follows.

$$
\left\{\begin{array}{l}
U_{\mathrm{s} 0}=\frac{Z_{\mathrm{s}}}{Z_{0}+Z_{\mathrm{s}}} U_{0}+\frac{\mathrm{j} X_{\mathrm{m}} Z_{0}}{Z_{0}+Z_{\mathrm{s}}} I_{\mathrm{r} 0} \\
U_{\mathrm{f} 0}=\frac{Z_{1}+Z_{\mathrm{s}}}{Z_{0}+Z_{\mathrm{s}}} U_{0}+\frac{\mathrm{j} X_{\mathrm{m}} Z_{2}}{Z_{0}+Z_{\mathrm{s}}} I_{\mathrm{r} 0}
\end{array}\right.
$$

where $Z_{\mathrm{s}}=R_{\mathrm{s}}+j \omega_{\mathrm{e}} L_{\mathrm{s}}, X_{\mathrm{m}}=\omega_{\mathrm{e}} L_{\mathrm{m}}, Z_{0}=Z_{1}+Z_{2}$.

It can seen that the steady-state component of terminal voltage is determined by the impedance of stator $Z_{\mathrm{s}}$, the impedance of transmission line $Z_{0}$, the mutual impedance $X_{\mathrm{m}}$, external grid voltage $U_{0}$ and rotor current $I_{\mathrm{r} 0}$ according to (3).

The rotor current is equal to rotor current reference value $\left(I_{\mathrm{r} 0}=I_{\mathrm{ref0} 0}\right)$ in the steady-state condition, which can be given by

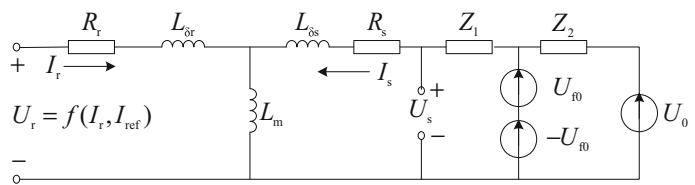

(a) Complete equivalent circuit of grid during grid fault

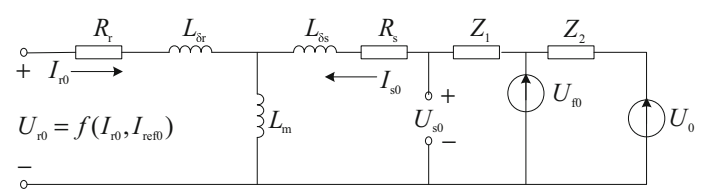

(b) Equivalent circuit of grid undernormal operation

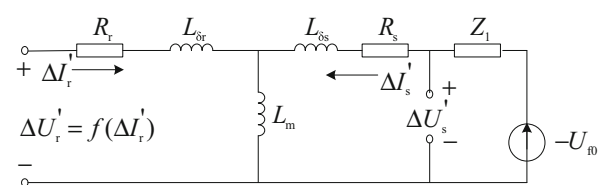

(c) Zero-state response equivalent circuit of $-U_{\mathrm{f} 0}$ ignoring rotor excitation control

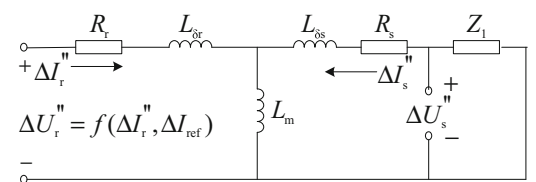

(d) Zero-state response equivalent circuit of control response fault component

Fig. 3 Application of superposition principle under grid fault

$$
\left\{\begin{array}{l}
I_{\text {rd_ref0 }}=-\frac{2 P_{\mathrm{s}} L_{\mathrm{s}}}{3 U_{\mathrm{s} 0} L_{\mathrm{m}}} \\
I_{\text {rd_ref0 }}=\frac{2 Q_{\mathrm{s}} L_{\mathrm{s}}}{3 U_{\mathrm{s} 0} L_{\mathrm{m}}}-\frac{U_{\mathrm{s} 0}}{\omega_{\mathrm{e}} L_{\mathrm{m}}} \\
I_{\text {ref0 }}=I_{\text {rd } \_ \text {ref0 } 0}+\mathrm{j} I_{\mathrm{rd} \_ \text {ref0 }}
\end{array}\right.
$$

\subsection{Zero-state response of grid fault voltage component}

The relationship between flux, voltage and current of wind power and power system can be defined as follows when consider alone the grid fault voltage component which was described in (1)-(3) and Fig. 3c.

$$
\left\{\begin{array}{l}
\Delta U_{\mathrm{s}}^{\prime}=\frac{\mathrm{d} \Delta \psi_{\mathrm{s}}^{\prime}}{\mathrm{d} t}+\mathrm{j} \omega_{\mathrm{r}} \Delta \psi_{\mathrm{s}}^{\prime}+R_{\mathrm{s}} \Delta I_{\mathrm{s}}^{\prime} \\
\Delta U_{\mathrm{r}}^{\prime}=\frac{\mathrm{d} \Delta \psi_{\mathrm{r}}^{\prime}}{\mathrm{d} t}+R_{\mathrm{r}} \Delta I_{\mathrm{r}}^{\prime} \\
\Delta \psi_{\mathrm{s}}^{\prime}=L_{\mathrm{s}} \Delta I_{\mathrm{s}}^{\prime}+L_{\mathrm{m}} \Delta I_{\mathrm{r}}^{\prime} \\
\Delta \psi_{\mathrm{r}}^{\prime}=L_{\mathrm{r}} \Delta I_{\mathrm{r}}^{\prime}+L_{\mathrm{m}} \Delta I_{\mathrm{s}}^{\prime} \\
-U_{\mathrm{f} 0}=\Delta U_{\mathrm{s}}^{\prime}+Z_{1} \Delta I_{\mathrm{s}}^{\prime} \\
\Delta U_{\mathrm{r}}^{\prime}=-K_{\mathrm{p}} \Delta I_{\mathrm{r}}^{\prime}-K_{\mathrm{i}} \int \Delta I_{\mathrm{r}}^{\prime} \mathrm{d} t+\mathrm{j} \omega \delta L_{\mathrm{r}} \Delta I_{\mathrm{r}}^{\prime}
\end{array}\right.
$$

The relationship between voltage, current and flux can be defined by the following equation in Laplace domain after the Laplace transformation of (5). 


$$
\left\{\begin{array}{l}
\Delta U_{\mathrm{s}}^{\prime}(s)=-U_{\mathrm{f} 0}(s)+Z_{1} \frac{f_{1}^{\prime}(s)}{L_{\mathrm{s}} L_{\mathrm{r}} \delta\left(s-s_{1}\right)\left(s-s_{2}\right)\left(s-s_{3}\right)} U_{\mathrm{f} 0}(s) \\
\Delta U_{\mathrm{r}}^{\prime}(s)=\frac{L_{\mathrm{m}} f_{2}^{\prime}(s)}{L_{\mathrm{s}} L_{\mathrm{r}} \delta\left(s-s_{1}\right)\left(s-s_{2}\right)\left(s-s_{3}\right)} U_{\mathrm{f} 0}(s) \\
\Delta \psi_{\mathrm{s}}^{\prime}(s)=-\frac{f_{3}^{\prime}(s)}{L_{\mathrm{r}} \delta\left(s-s_{1}\right)\left(s-s_{2}\right)\left(s-s_{3}\right)} U_{\mathrm{f} 0}(s) \\
\Delta \psi_{\mathrm{r}}^{\prime}(s)=-\frac{L_{\mathrm{m}} f_{4}^{\prime}(s)}{L_{\mathrm{s}} L_{\mathrm{r}} \delta\left(s-s_{1}\right)\left(s-s_{2}\right)\left(s-s_{3}\right)} U_{\mathrm{f} 0}(s), \\
\Delta I_{\mathrm{r}}^{\prime}(s)=\frac{L_{\mathrm{m}} f_{6}^{\prime}(s)}{L_{\mathrm{s}} L_{\mathrm{r}} \delta\left(s-s_{1}\right)\left(s-s_{2}\right)\left(s-s_{3}\right)} U_{\mathrm{f} 0}(s)
\end{array}\right.
$$

$$
\left\{\begin{array}{l}
f_{1}^{\prime}(s)=L_{\mathrm{r}} s^{2}+\left(R_{\mathrm{r}}+\mathrm{j} \omega L_{\mathrm{m}}^{2} / L_{\mathrm{s}}+K_{\mathrm{p}}\right) s+K_{\mathrm{i}} \\
f_{2}^{\prime}(s)=\left(-K_{\mathrm{p}} s-K_{\mathrm{i}}+\mathrm{j} \omega \delta L_{\mathrm{r}} s\right)(s+\mathrm{j} \omega) \\
f_{3}^{\prime}(s)=L_{\mathrm{r}} \delta s^{2}+\left(R_{r}+K_{\mathrm{p}}\right) s+K_{\mathrm{i}} \\
f_{4}^{\prime}(s)=\left(R_{\mathrm{r}}+K_{\mathrm{p}}-\mathrm{j} \omega \delta L_{\mathrm{r}}\right) s+K_{\mathrm{i}} \\
f_{5}^{\prime}(s)=L_{\mathrm{r}} s^{2}+\left(R_{\mathrm{r}}+\mathrm{j} \omega L_{\mathrm{r}}+K_{\mathrm{p}}-\mathrm{j} \omega \delta L_{\mathrm{r}}\right) s+K_{\mathrm{i}} \\
f_{6}^{\prime}(s)=s(s+\mathrm{j} \omega)
\end{array}\right.
$$

where $s_{1}, s_{2}, s_{3}$ are the solutions to the eigenvalue which can be written as

$$
\begin{aligned}
G(s)= & \left(L_{\mathrm{s}} L_{\mathrm{r}}-L_{\mathrm{m}}^{2}\right) s^{3}+\left[L_{\mathrm{s}}\left(R_{\mathrm{r}}+K_{\mathrm{p}}\right)+L_{\mathrm{r}}\left(R_{\mathrm{s}}+Z_{1}\right)\right. \\
& \left.+\mathrm{j} \omega_{\mathrm{e}}\left(L_{\mathrm{s}} L_{\mathrm{r}}-L_{\mathrm{m}}^{2}\right)\right] s^{2}+\left[K_{\mathrm{i}} L_{\mathrm{s}}+\left(R_{\mathrm{s}}+Z_{1}+\mathrm{j} \omega_{\mathrm{e}} L_{\mathrm{s}}\right)\right. \\
& \left.\times\left(R_{\mathrm{r}}+K_{\mathrm{p}}+\mathrm{j} \omega \frac{L_{\mathrm{m}}^{2}}{L_{\mathrm{s}}}\right)+L_{\mathrm{m}}^{2} \omega_{\mathrm{e}} \omega\right] s+\left(R_{\mathrm{s}}+Z_{1}+\mathrm{j} \omega_{\mathrm{e}} L_{\mathrm{s}}\right) K_{\mathrm{i}}
\end{aligned}
$$

According to (6), the time domain equation of voltage, current and flux based on zero-state response of grid fault voltage component can be expressed as follows.$$
\left\{\begin{array}{l}
\Delta U_{\mathrm{s}}^{\prime}=-U_{\mathrm{f} 0}+\frac{Z_{1} U_{\mathrm{f} 0}}{L_{\mathrm{s}} L_{\mathrm{r}} \delta}\left(\frac{f_{1}^{\prime}(0)}{A}+\frac{f_{1}^{\prime}\left(s_{1}\right)}{B} \mathrm{e}^{s_{1} t}+\frac{f_{1}^{\prime}\left(s_{2}\right)}{C} \mathrm{e}^{s_{2} t}+\frac{f_{1}^{\prime}\left(s_{3}\right)}{D} \mathrm{e}^{s_{3} t}\right) \\
\Delta U_{\mathrm{r}}^{\prime}=\frac{L_{\mathrm{m}} U_{\mathrm{f} 0}}{L_{\mathrm{s}} L_{\mathrm{r}} \delta}\left(\frac{f_{2}^{\prime}(0)}{A}+\frac{f_{2}^{\prime}\left(s_{1}\right)}{B} \mathrm{e}^{s_{1} t}+\frac{f_{2}^{\prime}\left(s_{2}\right)}{C} \mathrm{e}^{s_{2} t}+\frac{f_{2}^{\prime}\left(s_{3}\right)}{D} \mathrm{e}^{s_{3} t}\right) \\
\Delta \psi_{\mathrm{s}}^{\prime}=-\frac{U_{\mathrm{f} 0}}{L_{\mathrm{r}} \delta}\left(\frac{f_{3}^{\prime}(0)}{A}+\frac{f_{3}^{\prime}\left(s_{1}\right)}{B} \mathrm{e}^{s_{1} t}+\frac{f_{3}^{\prime}\left(s_{2}\right)}{C} \mathrm{e}^{s_{2} t}+\frac{f_{3}^{\prime}\left(s_{3}\right)}{D} \mathrm{e}^{s_{3} t}\right) \\
\Delta \psi_{\mathrm{r}}^{\prime}=-\frac{L_{\mathrm{m}} U_{\mathrm{f} 0}}{L_{\mathrm{s}} L_{\mathrm{r}} \delta}\left(\frac{f_{4}^{\prime}(0)}{A}+\frac{f_{4}^{\prime}\left(s_{1}\right)}{B} \mathrm{e}^{s_{1} t}+\frac{f_{4}^{\prime}\left(s_{2}\right)}{C} \mathrm{e}^{s_{2} t}+\frac{f_{4}^{\prime}\left(s_{3}\right)}{D} \mathrm{e}^{s_{3} t}\right) \\
\Delta I_{\mathrm{s}}^{\prime}=-\frac{U_{\mathrm{f} 0}}{L_{\mathrm{s}} L_{\mathrm{r}} \delta}\left(\frac{f_{5}^{\prime}(0)}{A}+\frac{f_{5}^{\prime}\left(s_{1}\right)}{B} \mathrm{e}^{s_{1} t}+\frac{f_{5}^{\prime}\left(s_{2}\right)}{C} \mathrm{e}^{s_{2} t}+\frac{f_{5}^{\prime}\left(s_{3}\right)}{D} \mathrm{e}^{s_{3} t}\right) \\
\Delta I_{\mathrm{r}}^{\prime}=\frac{L_{\mathrm{m}} U_{\mathrm{f} 0}}{L_{\mathrm{s}} L_{\mathrm{r}} \delta}\left(\frac{f_{6}^{\prime}(0)}{A}+\frac{f_{6}^{\prime}\left(s_{1}\right)}{B} \mathrm{e}^{s_{1} t}+\frac{f_{6}^{\prime}\left(s_{2}\right)}{C} \mathrm{e}^{s_{2} t}+\frac{f_{6}^{\prime}\left(s_{3}\right)}{D} \mathrm{e}^{s_{3} t}\right)
\end{array}\right.
$$

$$
\left\{\begin{array}{l}
A=-s_{1} s_{2} s_{3} \\
B=s_{1}\left(s_{1}-s_{2}\right)\left(s_{1}-s_{3}\right) \\
C=s_{2}\left(s_{2}-s_{1}\right)\left(s_{2}-s_{3}\right) \\
D=s_{3}\left(s_{3}-s_{1}\right)\left(s_{3}-s_{2}\right)
\end{array}\right.
$$

The grid fault voltage component $U_{\text {fo }}$ can be calculated by (3).
According to the analysis of zero-state response of grid fault voltage component $U_{\mathrm{fo}}$, we can see that the transient component $\Delta U^{\prime}$ s of terminal voltage consists of two steadystate components and three attenuation components from (9).

\subsection{Zero-state response of control response fault component}

When a grid fault occurs, the control strategies of DFIGs will change from normal mode to FRT mode. Then the control response fault component can be calculated by

$\Delta I_{\text {ref }}=-\left(I_{\text {ref } 0}-I_{\text {refl } 1}\right)$

where $I_{\text {ref1 }}$ is the reference value of rotor current under FRT control strategy, which can be calculated as

$$
\left\{\begin{array}{l}
I_{\text {rd_ref1 }}=\lim \left(0, \sqrt{I_{\max }^{2}-I_{\text {rd_ref1 }}^{2}}\right) \\
I_{\text {rd_ref1 }}=-U_{\mathrm{T}} / \omega_{\mathrm{e}} L_{\mathrm{m}}-K\left(0.9-U_{\mathrm{T}}\right) I_{\mathrm{N}} L_{\mathrm{s}} / L_{\mathrm{m}} \\
I_{\text {ref1 }}=I_{\text {rd_ref1 }}+\mathrm{j} I_{\text {rd_ref1 }}
\end{array}\right.
$$

where $I_{N}$ is the rated current of DFIGs; $U_{\mathrm{T}}$ is the voltage of the point of common coupling (PCC); $K$ is the dynamic reactive power compensation coefficient; $I_{\max }$ is the maximum rotor current.

Zero-state response of control response fault component based on (1), (2) and Fig. 3d can be calculated as

$$
\left\{\begin{array}{l}
\Delta U_{\mathrm{s}}^{\prime \prime}=\frac{\mathrm{d} \Delta \psi_{\mathrm{s}}^{\prime \prime}}{\mathrm{d} t}+\mathrm{j} \omega_{\mathrm{e}} \Delta \psi_{\mathrm{s}}^{\prime \prime}+R_{\mathrm{s}} \Delta I_{\mathrm{s}}^{\prime \prime} \\
\Delta U_{\mathrm{r}}^{\prime \prime}=\frac{\mathrm{d} \Delta \psi_{\mathrm{r}}^{\prime \prime}}{\mathrm{d} t}+\mathrm{j} \omega \Delta \psi_{\mathrm{r}}^{\prime \prime}+R_{\mathrm{r}} \Delta I_{\mathrm{r}}^{\prime \prime} \\
\Delta \psi_{\mathrm{s}}^{\prime \prime}=L_{\mathrm{s}} \Delta I_{\mathrm{s}}^{\prime \prime}+L_{\mathrm{m}} \Delta I_{\mathrm{r}}^{\prime \prime} \\
\Delta \psi_{\mathrm{r}}^{\prime \prime}=L_{\mathrm{r}} \Delta I_{\mathrm{r}}^{\prime \prime}+L_{\mathrm{m}} \Delta I_{\mathrm{s}}^{\prime \prime} \\
0=\Delta U_{s}^{\prime \prime}+Z_{1} \Delta I_{s}^{\prime \prime} \\
\Delta U_{\mathrm{r}}^{\prime \prime}=K_{\mathrm{p}}\left(\Delta I_{\mathrm{ref}}-\Delta I_{\mathrm{r}}^{\prime \prime}\right) \\
\quad+K_{\mathrm{i}} \int\left(\Delta I_{\mathrm{ref}}-\Delta I_{\mathrm{r}}^{\prime \prime}\right) \mathrm{d} t+\mathrm{j} \omega \delta L_{\mathrm{r}} \Delta I_{\mathrm{r}}^{\prime \prime}
\end{array}\right.
$$

The relationship between voltage, current and flux in Laplace domain based on (13) are defined as

$$
\left\{\begin{array}{l}
\Delta U_{\mathrm{s}}^{\prime \prime}(s)=\frac{Z_{1} L_{\mathrm{m}} f_{1}^{\prime \prime}(s)}{L_{\mathrm{s}} L_{\mathrm{r}} \delta\left(s-s_{1}\right)\left(s-s_{2}\right)\left(s-s_{3}\right)} \Delta I_{\mathrm{ref}}(s) \\
\Delta U_{\mathrm{r}}^{\prime \prime}(s)=\frac{f_{2}^{\prime \prime}(s)}{L_{\mathrm{s}} L_{\mathrm{r}} \delta\left(s-s_{1}\right)\left(s-s_{2}\right)\left(s-s_{3}\right)} \Delta I_{\mathrm{ref}}(s) \\
\Delta \psi_{\mathrm{s}}^{\prime \prime}(s)=\frac{L_{\mathrm{m}} f_{3}^{\prime \prime}(s)}{L_{\mathrm{s}} L_{\mathrm{r}} \delta\left(s-s_{1}\right)\left(s-s_{2}\right)\left(s-s_{3}\right)} \Delta I_{\mathrm{ref}}(s) \\
\Delta \psi_{\mathrm{r}}^{\prime \prime}(s)=\frac{f_{4}^{\prime \prime}(s)}{L_{s} L_{\mathrm{r}} \delta\left(s-s_{1}\right)\left(s-s_{2}\right)\left(s-s_{3}\right)} \Delta I_{\mathrm{ref}}(s) \\
\Delta I_{\mathrm{s}}^{\prime \prime}(s)=-\frac{L_{\mathrm{m}} f_{5}^{\prime \prime}(s)}{L_{\mathrm{s}} L_{\mathrm{r}} \delta\left(s-s_{1}\right)\left(s-s_{2}\right)\left(s-s_{3}\right)} \Delta I_{\mathrm{ref}}(s) \\
\Delta I_{\mathrm{r}}^{\prime \prime}(s)=\frac{f_{6}^{\prime \prime}(s)}{L_{\mathrm{s}} L_{\mathrm{r}} \delta\left(s-s_{1}\right)\left(s-s_{2}\right)\left(s-s_{3}\right)} \Delta I_{\mathrm{ref}}(s)
\end{array}\right.
$$




$$
\left\{\begin{array}{l}
f_{1}^{\prime \prime}(s)=\left(s+\mathrm{j} \omega_{\mathrm{e}}\right)\left(K_{\mathrm{p}} s+K_{\mathrm{i}}\right) \\
f_{2}^{\prime \prime}(s)=\left[\left(\mathrm{j} \omega L_{\mathrm{r}} \delta+R_{\mathrm{r}}\right) L_{\mathrm{s}} s+Z_{\mathrm{r}} Z_{\mathrm{s}}^{\prime}+\omega \omega_{\mathrm{e}} L_{\mathrm{m}}^{2}\right]\left(K_{\mathrm{p}} s+K_{\mathrm{i}}\right) \\
f_{3}^{\prime \prime}(s)=\left(R_{\mathrm{s}}+Z_{1}\right)\left(K_{\mathrm{p}} s+K_{\mathrm{i}}\right) \\
f_{4}^{\prime \prime}(s)=\left[L_{\mathrm{s}} L_{\mathrm{r}} \delta s+L_{\mathrm{r}}\left(R_{\mathrm{s}}+Z_{1}\right)+\mathrm{j} \omega_{\mathrm{e}} L_{\mathrm{s}} L_{\mathrm{r}} \delta\right]\left(K_{\mathrm{p}} s+K_{\mathrm{i}}\right) \\
f_{5}^{\prime \prime}(s)=\left(s+\mathrm{j} \omega_{\mathrm{e}}\right)\left(K_{\mathrm{p}} s+K_{\mathrm{i}}\right) \\
f_{6}^{\prime \prime}(s)=\left(R_{\mathrm{s}}+Z_{1}+L_{\mathrm{s}} s+\mathrm{j} \omega_{\mathrm{e}} L_{\mathrm{s}}\right)\left(K_{\mathrm{p}} s+K_{\mathrm{i}}\right)
\end{array}\right.
$$

According to (14), the time domain equation of voltage, current and flux based on zero-state response of control response fault component can be expressed as

$$
\left\{\begin{array}{l}
\Delta U_{\mathrm{s}}^{\prime \prime}=\frac{Z_{1} L_{\mathrm{m}} \Delta I_{\mathrm{ref}}}{L_{\mathrm{s}} L_{\mathrm{r}} \delta}\left(\frac{f_{1}^{\prime \prime}(0)}{A}+\frac{f_{1}^{\prime \prime}\left(s_{1}\right)}{B} \mathrm{e}^{s_{1} t}+\frac{f_{1}^{\prime \prime}\left(s_{2}\right)}{C} \mathrm{e}^{s_{2} t}+\frac{f_{1}^{\prime \prime}\left(s_{3}\right)}{D} \mathrm{e}^{s_{3} t}\right) \\
\Delta U_{\mathrm{r}}^{\prime \prime}=\frac{\Delta I_{\mathrm{ref}}}{L_{\mathrm{s}} L_{\mathrm{r}} \delta}\left(\frac{f_{2}^{\prime \prime}(0)}{A}+\frac{f_{2}^{\prime \prime}\left(s_{1}\right)}{B} \mathrm{e}^{s_{1} t}+\frac{f_{2}^{\prime \prime}\left(s_{2}\right)}{C} \mathrm{e}^{s_{2} t}+\frac{f_{2}^{\prime \prime}\left(s_{3}\right)}{D} \mathrm{e}^{s_{3} t}\right) \\
\Delta \psi_{\mathrm{s}}^{\prime \prime}=\frac{L_{\mathrm{m}} \Delta I_{\mathrm{ref}}}{L_{\mathrm{s}} L_{\mathrm{r}} \delta}\left(\frac{f_{3}^{\prime \prime}(0)}{A}+\frac{f_{3}^{\prime \prime}\left(s_{1}\right)}{B} \mathrm{e}^{s_{1} t}+\frac{f_{3}^{\prime \prime}\left(s_{2}\right)}{C} \mathrm{e}^{s_{2} t}+\frac{f_{3}^{\prime \prime}\left(s_{3}\right)}{D} \mathrm{e}^{s_{3} t}\right) \\
\Delta \psi_{\mathrm{r}}^{\prime \prime}=\frac{\Delta I_{\mathrm{ref}}}{L_{\mathrm{s}} L_{\mathrm{r}} \delta}\left(\frac{f_{4}^{\prime \prime}(0)}{A}+\frac{f_{4}^{\prime \prime}\left(s_{1}\right)}{B} \mathrm{e}^{s_{1} t}+\frac{f_{4}^{\prime \prime}\left(s_{2}\right)}{C} \mathrm{e}^{s_{2} t}+\frac{f_{4}^{\prime \prime}\left(s_{3}\right)}{D} \mathrm{e}^{s_{3} t}\right) \\
\Delta I_{\mathrm{s}}^{\prime \prime}=-\frac{L_{\mathrm{m}} \Delta I_{\mathrm{ref}}}{L_{\mathrm{s}} L_{\mathrm{r}} \delta}\left(\frac{f_{5}^{\prime \prime}(0)}{A}+\frac{f_{5}^{\prime \prime}\left(s_{1}\right)}{B} \mathrm{e}^{s_{1} t}+\frac{f_{5}^{\prime \prime}\left(s_{2}\right)}{C} \mathrm{e}^{s_{2} t}+\frac{f_{5}^{\prime \prime}\left(s_{3}\right)}{D} \mathrm{e}^{s_{3} t}\right) \\
\Delta I_{\mathrm{r}}^{\prime \prime}=\frac{\Delta I_{\mathrm{ref}}}{L_{\mathrm{s}} L_{\mathrm{r}} \delta}\left(\frac{f_{6}^{\prime \prime}(0)}{A}+\frac{f_{6}^{\prime \prime}\left(s_{1}\right)}{B} \mathrm{e}^{s_{1} t}+\frac{f_{6}^{\prime \prime}\left(s_{2}\right)}{C} \mathrm{e}^{s_{2} t}+\frac{f_{6}^{\prime \prime}\left(s_{3}\right)}{D} \mathrm{e}^{s_{3} t}\right)
\end{array}\right.
$$

According to the analysis of zero-state response of control response fault component, we can see that the transient component $\Delta U_{\mathrm{s}}^{\prime}$ of terminal voltage consists of one steady-state component and three attenuation components from (16).

\section{Analysis on voltage phase angle jump under grid fault in case of connecting DFIGs into weak grid}

Based on the above analysis, the terminal voltage of DFIGs when a grid fault occurs based on (3), (9) and (16) can be expressed as

$$
\begin{aligned}
U_{\mathrm{s}}= & U_{\mathrm{s} 0}+\Delta U_{\mathrm{s}}^{\prime}+\Delta U_{\mathrm{s}}^{\prime \prime}=U_{\mathrm{s} 0}^{\prime}+U_{\mathrm{s} 1}^{\prime}+U_{\mathrm{s} 2}^{\prime} \\
& +U_{\mathrm{s} 3}^{\prime}+U_{\mathrm{s} 1}^{\prime \prime}+U_{\mathrm{s} 2}^{\prime \prime}+U_{\mathrm{s} 3}^{\prime \prime}=\left|U_{\mathrm{s}}\right| \angle \theta_{1}
\end{aligned}
$$

Each component value can be calculated by

$$
\left\{\begin{array}{l}
U_{\mathrm{s} 0}^{\prime}=U_{\mathrm{s} 0}-U_{\mathrm{f} 0}+\frac{Z_{1} f_{1}^{\prime}(0)}{L_{\mathrm{s}} L_{\mathrm{r}} \delta A} U_{\mathrm{f} 0}+\frac{Z_{1} L_{\mathrm{m}} f_{1}^{\prime \prime}(0)}{L_{\mathrm{s}} L_{\mathrm{r}} \delta A} \Delta I_{\mathrm{ref}} \\
U_{\mathrm{s} 1}^{\prime}=\left[f_{1}^{\prime}\left(s_{1}\right) U_{\mathrm{f} 0}+L_{\mathrm{m}} f_{1}^{\prime \prime}\left(s_{1}\right) \Delta I_{\mathrm{ref}}\right] \frac{Z_{1}}{L_{\mathrm{s}} L_{\mathrm{r}} \delta B} \mathrm{e}^{s_{1} t} \\
U_{\mathrm{s} 2}^{\prime}=\left[f_{1}^{\prime}\left(s_{2}\right) U_{\mathrm{f} 0}+L_{\mathrm{m}} f_{1}^{\prime \prime}\left(s_{2}\right) \Delta I_{\mathrm{ref}}\right] \frac{Z_{1}}{L_{\mathrm{s}} L_{\mathrm{r}} \delta C} \mathrm{e}^{s_{2} t} \\
U_{\mathrm{s} 3}^{\prime}=\left[f_{1}^{\prime}\left(s_{3}\right) U_{\mathrm{f} 0}+L_{\mathrm{m}} f_{1}^{\prime \prime}\left(s_{3}\right) \Delta I_{\mathrm{ref}}\right] \frac{Z_{1}}{L_{\mathrm{s}} L_{\mathrm{r}} \delta D} \mathrm{e}^{s_{3} t}
\end{array}\right.
$$

where $U_{\mathrm{s} 0}^{\prime}$ is the steady-state component of terminal voltage during the grid fault; $U_{\mathrm{s} 1}^{\prime}, U_{\mathrm{s} 2}^{\prime}, U_{\mathrm{s} 3}^{\prime}$ are the transient components of terminal voltage.

According to (17) and (18), the terminal voltage vector diagram of DFIGs during the grid fault is presented in Fig. 4. In Fig. 4,

$$
\left\{\begin{array}{l}
\Delta U_{1}=\frac{Z_{1} f_{1}^{\prime}(0)}{L_{\mathrm{s}} L_{\mathrm{r}} \delta A} U_{\mathrm{f} 0} \\
\Delta U_{2}=\frac{Z_{1} L_{\mathrm{m}} f_{1}^{\prime \prime}(0)}{L_{\mathrm{s}} L_{\mathrm{r}} \delta A} \Delta I_{\mathrm{ref}}
\end{array}\right.
$$

Equations (3), (18), (19) and Fig. 4 show that the transient characteristics of terminal voltage are associated with control strategies of DFIGs, control parameters of current inner-loop of rotor-side converter and grid strength. The following conclusions can be drawn.

1) The transient behavior of internal voltage phase angle of DFIGs is different from conventional synchronous generators, not depending on the rotor position, and showing an electrical feature. The terminal voltage phase angle of DFIGs jumps when a grid fault occurs.

2) The jump value of terminal voltage phase angle is described in (20). The main influencing factor is the rotor excitation control current according to (18)-(19). The bigger reactive power compensation current, the smaller jump value of terminal voltage phase angle.

$\Delta \theta=\theta_{2}-\theta_{0}$

3) The change of terminal voltage phase angle is an electromagnetic transient process when a grid fault occurs, the jump rate is determined by the decay time constant $s_{1}, s_{2}$ and $s_{3}$ according to (18. According to (8), the decay time constant $s_{1}$, $s_{2}$ and $s_{3}$ are functions of control parameters of current innerloop of rotor-side converter, and the decay time constant is about several tens of milliseconds. The larger control parameters $\left(K_{\mathrm{p}}\right.$ or $\left.K_{\mathrm{i}}\right)$ of current inner-loop of rotor-side converter, the bigger jump rate of terminal voltage phase angle.

4) Considering the most serious condition, the DFIGs and grid are separated at fault location during the grid fault when a three-phase short circuit fault occurs. According to (3) and (18), the relative jump value and jump rate of terminal voltage phase angle are not related with the impedance of line $Z_{0}$, but the initial terminal voltage phase angle is related with the

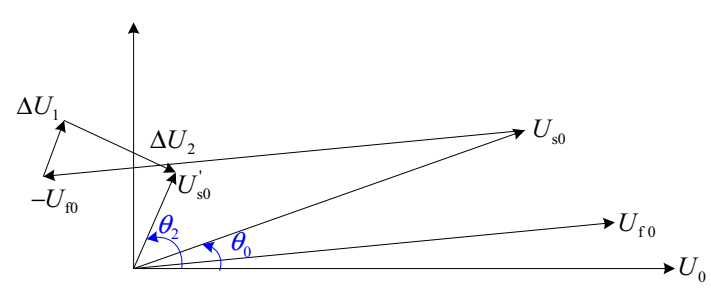

Fig. 4 Terminal voltage vector diagram of DFIGs during grid fault 


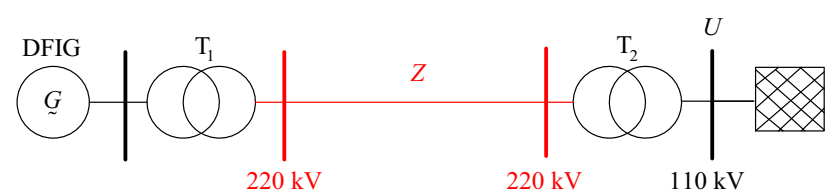

Fig. 5 Structure diagram of simulation system

impedance of line. The larger impedance of line, the bigger absolute value of terminal voltage phase angle. And the fault location mainly influences the voltage drop amplitude.

\section{Examples and analysis}

As analyzed in Section 4, the terminal voltage phase angle of DFIGs jumps when a grid fault occurs in the condition of connecting large scale DFIGs into weak grid. In order to investigate the accuracy of the theoretical analysis in this paper, an example is provided in this section. Fig. 5 shows the study system, which includes one hundred 1.5 MW DFIGs. The parameters obtained from an actual operation of DFIGs are adopted, i.e., $U_{\mathrm{N}}=690 \mathrm{~V}$, $R_{\mathrm{S}}=0.01, R_{\mathrm{r}}=0.01, L_{\mathrm{S}}=0.1, L_{\mathrm{r}}=0.1, L_{\mathrm{m}}=3.5$. Wind farms are connected to infinite power grid through one hundred kilometers single circuit transmission line. The resistance of the line is $0.04 \Omega / \mathrm{km}$, and the inductance of the line is $0.41 \mathrm{mH} / \mathrm{km}$. Supposing the crowbar protection is not invested (considered), and the DFIGs is controlled during the grid fault.

Figure 6 gives the simulation results and field data curves of DFIGs when the terminal voltage dropped to 0.2 and lasted for $625 \mathrm{~ms}$. The FRT field data comes from grid connected 1.5 MW DFIGs manufactured by Dongfang Turbine Co., Ltd. The simulation results are almost consistent with the field data during the grid fault from the curves, and the terminal voltage phase angle suddenly changes when a grid fault occurs.

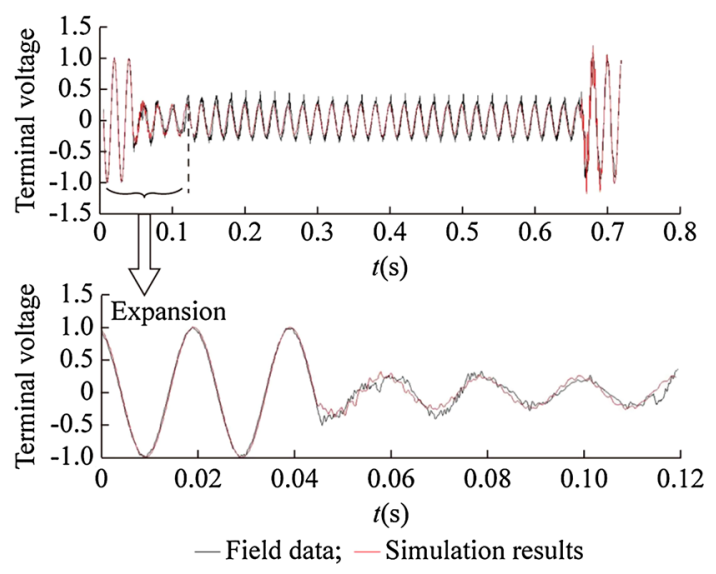

Fig. 6 Simulation results and field data
Assuming there is a three-phase short circuit fault in transmission line, and the fault is cleared after 120 milliseconds. Four comparison experiments are designed to analyze the transient characteristics of terminal voltage phase angle of DFIGs and its influencing factors.

\subsection{Analysis on transient characteristics of terminal voltage phase angle of DFIGs and synchronous generator}

In order to analyze the difference of transient characteristics of terminal voltage phase angle between DFIGs and synchronous generator, the wind farms based on DFIGs are replaced by a synchronous generator in Fig. 5 . The synchronous generator model includes sixth order generator model, governor model and regulator model. The classical parameters of synchronous generator are used, and the rated power of which is $150 \mathrm{MW}$. Figure 7 shows the terminal voltage phase angle curves of these two machines under grid fault.

Figure 7 shows that the terminal voltage phase angle of DFIGs jumps, and the jump value is bigger when a grid fault occurs or the grid fault is cleared. The change of terminal voltage phase angle of synchronous generator is slow because of the mechanical inertia during the grid fault, and it becomes oscillation attenuation after clearing the grid fault.

\subsection{Analysis of influence of FRT control strategies to terminal voltage phase angle}

Different FRT control strategies of DFIGs are adopted during the grid fault through giving different dynamic reactive power compensation coefficient $K$, the comparison curves of terminal voltage phase angle are shown in Fig. 8.

Figure 8 shows that the maximum jump value of terminal voltage phase angle is $57.1^{\circ}$ when $K=1.5$. The maximum jump value of terminal voltage phase angle is

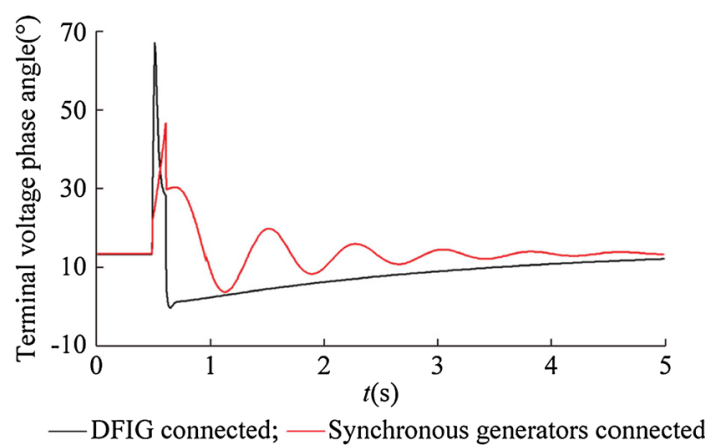

Fig. 7 Terminal voltage phase angle of DFIGs and synchronous generator 


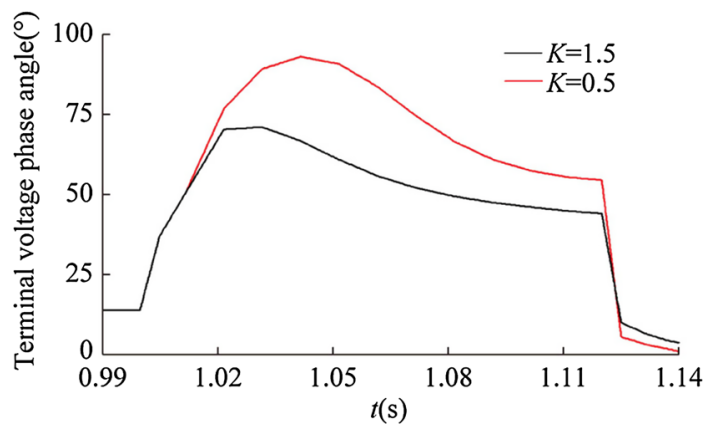

Fig. 8 Terminal voltage phase angle under different FRT control strategies

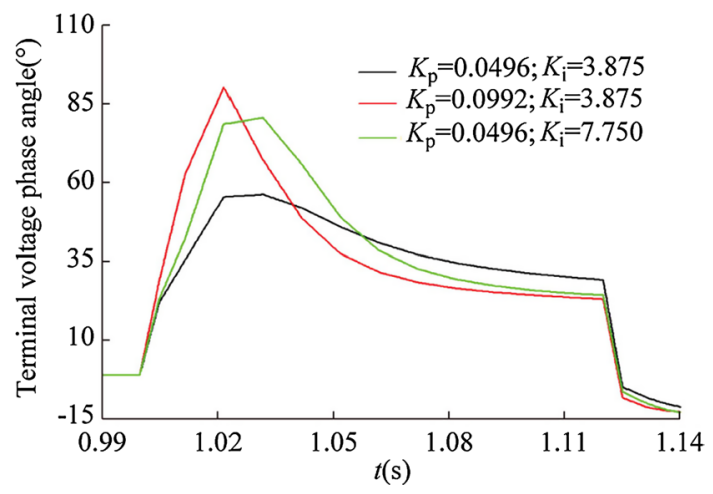

Fig. 9 Terminal voltage phase angle under different control parameters of current inner-loop of rotor-side converter

$79.2^{\circ}$ when $K=0.5$. The jump rate of terminal voltage phase angle is always $2.6 \% \mathrm{~ms}$ based on different dynamic reactive power compensation coefficient. So a big dynamic reactive power compensation coefficient can reduce maximum jump value and steady-state value of terminal voltage phase angle, and the jump rate of terminal voltage phase angle is unaffected.

\subsection{Analysis of influence of control parameters of current inner-loop of rotor-side converter to terminal voltage phase angle}

Different control parameters of current inner-loop of rotor-side converter are adopted during the grid fault, the comparison curves of terminal voltage phase angle are shown in Fig. 9.

Figure 9 shows that the jump rate of terminal voltage phase angle is $2.6 \% \mathrm{~ms}$, and the maximum jump value of terminal voltage phase angle is $57.1^{\circ}$ when the control parameters of current inner-loop of rotor-side converter are set as $K_{\mathrm{p}}=0.0496, K_{\mathrm{i}}=3.875$. The jump rate of terminal voltage phase angle is $4.14 \% \mathrm{~ms}$, and the maximum jump value of terminal voltage phase angle is $91.1^{\circ}$ when the control parameters of current inner-loop of rotor-side

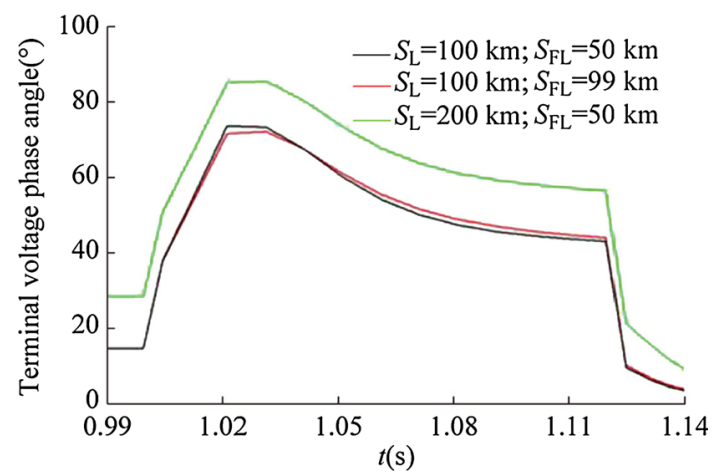

Fig. 10 Terminal voltage phase angle under different fault location and different grid strength

converter are set as $K_{\mathrm{p}}=0.0992, K_{\mathrm{i}}=3.875$. The jump rate of terminal voltage phase angle is $3.25 \% \mathrm{~ms}$, and the maximum jump value of terminal voltage phase angle is $71.4^{\circ}$ when the control parameters of current inner-loop of rotor-side converter are set as $K_{\mathrm{p}}=0.0496, K_{\mathrm{i}}=7.75$. So the small control parameters of current inner-loop of rotorside converter can reduce jump rate and maximum jump value of terminal voltage phase angle, and the steady-state value of terminal voltage phase angle is nearby.

\subsection{Analysis of influence of grid structure change to terminal voltage phase angle}

The comparison curves of terminal voltage phase angle are shown in Fig. 10 when a grid fault occurs based on different fault location $S_{\mathrm{FL}}$ and different grid strength $S_{\mathrm{L}}$.

Figure 10 shows that the jump rate of terminal voltage phase angle is always $2.6 \% \mathrm{~ms}$, and the maximum jump value of terminal voltage phase angle is almost $57.1^{\circ}$ based on different fault location and different grid strength. But the absolute value change of terminal voltage phase angle is bigger in case of connecting large scale DFIGs into weak grid.

\section{Conclusion}

The mechanism on voltage phase angle jump under grid fault considering weak grid with DFIGs integration has been present in this paper. Because of the application of non-synchronous generators (such as DFIGs), which are equipped with power electronics (such as converter), the characteristics of DFIGs and synchronous generator are different. The internal potential of DFIGs is not depend on the rotor position, it is electrical quantity rather than physical quantity, and it jumps when a grid fault occurs. The jump rate and maximum jump value of terminal 
voltage phase angle are mainly affected by the FRT control strategies and control parameters of current inner-loop of rotor-side converter. The grid strength affects the variation on absolute value of terminal voltage phase angle. This paper establishes theoretical foundation for the research about integrating large scale wind power into weak grid, and solves technical problems to guarantee safe operation of weak grid with large scale wind power integration.

Acknowledgment This work was supported by The National Basic Research Program (973 Program) 2012CB215105.

Open Access This article is distributed under the terms of the Creative Commons Attribution 4.0 International License (http:// creativecommons.org/licenses/by/4.0/), which permits unrestricted use, distribution, and reproduction in any medium, provided you give appropriate credit to the original author(s) and the source, provide a link to the Creative Commons license, and indicate if changes were made.

\section{References}

[1] China installed wind power capacity. Chinese Wind Energy Association (2014), Beijing, China, 2015 (in Chinese)

[2] He YK, Hu JB (2012) Several hot-spot issues associated with the grid-connected operations of wind-turbine driven doubly fed induction generators. P CSEE 32(27):1-15 (in Chinese)

[3] Yuan XM (2013) Overview of problems in large-scale wind integrations. J Mod Power Syst Clean Energ 1(1):22-25. doi:10. 1007/s40565-013-0010-6

[4] Liu QH, He YK, Zhang JH (2006) Operation control and modeling simulation of AC-excited variable-speed constantfrequency (AEVSCF) wind power generator. P CSE 26(5):43-50 (in Chinese)

[5] Chi YN, Wang WS, Liu YH et al (2006) Impact of large scale wind farm integration on power system transient stability. Automat Electr Power Syst 30(15):10-14 (in Chinese)

[6] Liu XG, Xu Z, Wong KP (2013) Recent advancement on technical requirements for grid integration of wind power. J Mod Power Syst Clean Energy 1(3):216-222. doi:10.1007/s40565013-0036-9

[7] Zhou NC, Xie GL, Wang QG, et al (2014) Evaluation of threephase short-circuit peak current for doubly-fed induction generators integrated into power grids. Automat Electr Power Syst 38(18):65-71 (in Chinese). doi:10.7500/AEPS20130925005

[8] Miao L, Fang JK, Wen JY et al (2013) Transient stability risk assessment of power systems incorporating wind farms. J Mod Power Syst Clean Energy 1(2):134-141. doi:10.1007/s40565013-0022-2

[9] Morren J, de Haan SWH (2007) Short-circuit current of wind turbines with doubly fed induction generator. IEEE Trans Energy Convers 22(1):174-180

[10] Yang SY, Sun DY, Chen LW et al (2013) Study on electromagnetic transition of DFIG-based wind turbines under grid fault based on analytical method. P CSEE 33(S1):13-20 (in Chinese)

[11] Kong XP, Zhang Z, Yin XG et al (2015) Study of fault current characteristics of DFIG considering impact of crowbar protection. Trans China Electrotechnol Soc 30(8):1-10 (in Chinese)
[12] Rahimi M, Parniani M (2010) Grid-fault ride-through analysis and control of wind turbines with doubly fed induction generators. Electr Power Syst Res 80(2):184-195

[13] Lopez J, Sanchis P, Roboam X et al (2007) Dynamic behavior of the doubly fed induction generator during three-phase voltage dips. IEEE Trans Energy Convers 22(3):709-717

[14] Tian XS, Wang WS, Chi YN et al (2015) Performances of DFIG-based wind turbines during system fault and its impact on transient stability of power system. Automat Electr Power Syst 39(10):16-21 (in Chinese). doi:10.7500/AEPS20140424006

[15] Gautam D, Vittal V, Harbour T (2009) Impact of increased penetration of DFIG-based wind turbine generators on transient and small signal stability of power systems. IEEE Trans Power Syst 24(3):1426-1434

[16] Vittal E, O'Malley M, Keane A (2012) Rotor angle stability with high penetrations of wind generation. IEEE Trans Power Syst 27(1):353-362

[17] Xiong XF, Ouyang JX, Wen A (2012) An analysis on impacts and characteristics of voltage of DFIG-based wind turbine generator under grid short circuit. Automat Electr Power Syst 36(14):143-149 (in Chinese)

[18] Wang W, Chen N, Zhu LZ et al (2009) Phase angle compensation control strategy for low-voltage ride through of doublyfed induction generator. P CSEE 29(21):62-68 (in Chinese)

[19] Hao ZH (2011) Transient behavior of DFIG and its influence on power system stability. Master Thesis, Tianjin University, Tianjin, China (in Chinese)

[20] Mohseni M, Islam S, Masoum MAS (2011) Impacts of symmetrical and asymmetrical voltage sags on DFIG-based wind turbines considering phase-angle jump, voltage recovery, and sag parameters. IEEE Trans Power Electron 26(5):1587-1598

[21] Wang Y, Wu QW (2014) Electromagnetic transient response analysis of DFIG under cascading grid faults considering phase angel jumps. In: Proceedings of the 17th international conference on electrical machines and systems (ICEMS'14), Hangzhou, China, 22-25 Oct 2014, pp 1340-1344

[22] Ma J, Lan XB, Ding XX et al (2014) Transient characteristics of symmetrical short circuit fault in double fed induction generators considering grid-side converter control and phase-angle jump of DFIG's terminal voltage. Power Syst Technol 38(7):1891-1897 (in Chinese)

Xinshou TIAN received his B.E. and M.E. degrees from Huazhong University of Science and Technology, China, in 2008, and China Electric Power Research Institute, China, in 2011, respectively. He is currently working toward the Ph.D degree in electrical engineering at North China Electric Power University, Beijing, China. His main research interests include wind power generation and power system stability analysis.

Gengyin LI received his B.S., M.S., and Ph.D degrees from North China Electric Power University, Beijing, China, in 1984, 1987, and 1996, respectively, all in electrical engineering. Since1987, he has been with the School of Electrical and Electronic Engineering, North China Electric Power University, Beijing, China, where he is currently a professor and executive dean of the school. His areas of interest include power system analysis and control, HVDC and VSCHVDC transmission technology.

Yongning CHI received his B.E. and M.E. degrees from Shandong University, China, in 1995, and 2002, respectively, and Ph.D degree in electrical engineering from China Electric Power Research Institute, China, in 2006. Since 2003 he has been employed at China 
Electric Power Research Institute (CEPRI), Beijing, China, where he is the chief engineer for Renewable Energy Department. His research of interests is in modeling, control and integration analysis of renewable energy generation.

Weisheng WANG received his B.E. and M.E. degrees from Xi'an University of Technology, China, in 1990, and 1993, and Ph.D degree in electrical engineering from Xi'an Jiaotong University, China, in 1996. Since 1997 he has been employed at China Electric Power Research Institute (CEPRI), Beijing, China, where he is the senior engineer (professor) and director of Renewable Energy Department of CEPRI. His research of interests is in integration of renewable energy generation.
Haiyan TANG received his B.E. and M.E. degrees from HeFei University of Technology, China, in 2000, and 2003, respectively, and Ph.D degree in electrical engineering from China Electric Power Research Institute, China, in 2008. Since 2008 he has been employed at China Electric Power Research Institute (CEPRI), Beijing, China. His research of interests is in modeling, control and integration analysis of renewable energy generation.

Xiang LI received her B.E. degree from Tsinghua University, China in 2009. She is currently working toward the M.E. degree in China Electric Power Research Institute since 1999. Her main research interests include wind power integration and stability analysis. 